\title{
Interpolation Algorithm for NURBS Curve with Scheduled Feedrate on Curvature Extreme
}

\author{
Guo-Shun JI ${ }^{\mathrm{a}, *}$, Wu-Jia YU ${ }^{\mathrm{b}}$, Zhi-Ping CHEN ${ }^{\mathrm{c}}$
}

School of Mechanical Engineering, Hangzhou Dianzi University, Hangzhou, Zhejiang, 310018, China

ajgsh@hdu.edu.cn

${ }^{*}$ Corresponding author

Keywords: NURBS curve interpolation, Curvature extreme, Scheduling feedrate.

Abstract. In CNC machining, it is desired to obtain high machining accuracy with high processing velocity as far as possible, however it is difficult to realize, and especially machining NURBS curve with large curvature. Based on scheduled feedrate on curvature of NURBS, a new NURBS curve interpolation algorithm was proposed. First, the curvature extreme of NURBS curve was calculated, and the critical value was defined according to machining dynamical requirements. Second, the NURBS curve was split several sub-segments, feedrate scanning algorithm based on $\mathrm{S}$ curve acceleration/deceleration was used to schedule the feedrate within each sub-segments and the connection points between adjacent sub-segments. Last, during real-time interpolation, with the second-order Taylor expansion, the interpolation parameter was computed. The results of interpolation case indicated the proposed method not obtain high interpolation accuracy with high interpolation feedrate, but the interpolation output acceleration and jerk within the machine tool's dynamical requirements.

\section{Introduction}

Pan Haihong et al. proposed a adaptive piecewise NURBS curve interpolator[1], the NURBS curve was split to several sub-segments according to its curvature, and 17 types ACC/DEC were applied to schedule the feedrate within each sub-segments NURBS curve but the feedrate transition between adjacent sub-segments was not discussed in[2]. Based on splitting NURBS curve in accordance with its curvature, another complete $\mathrm{S}$-shape feed rate scheduling approach for NURBS interpolator was established by Xu Du et al.[2]. However the identical scanning feedrate approach from one end feedrate to another end within each long and short sub-segment was applied, the scanned reasonable feedrate for two end feedrate of each sub-segments with different length were out of question, and the scheduled feedrate within each sub-segments were hard to be optimized as far as possible. The S-shape method is widely used in smooth feedrate scheduling because of its simplicity and smoothness[3], but the interpolation accuracy and fluctuation of output speed of interpolator were greatly affected by the accuracy of arc length. Aim at increase interpolation accuracy in high speed machining of NURBS curve, especially for the one with sharp corner, a new NURBS curve interpolation algorithm was proposed based on scheduled feedrate on curvature extreme of NURBS curve.

\section{NURBS curve and its arc length}

The $k$ order NURBS curve with $n+1$ control points can be expressed with the piecewise rational polynomial vector point function (1).

$$
\mathbf{p}(u)=\sum_{i=0}^{n} \omega_{i} \mathbf{d}_{i} N_{i, k}(u) / \sum_{i=0}^{n} \omega_{i} N_{i, k}(u)
$$


Where $\omega_{i}(i=0,1, \cdots n)$ are the weight factor, $\mathbf{d}_{i}(i=0,1, \cdots n)$ are the control points, $N_{i, k}(u)$, as expression (2), are the basic function constructing with the knot vector $\mathbf{U}=\left[\mathrm{u}_{0}, \cdots, u_{i}, \cdots, u_{n+k+1}\right]$.

$$
\left\{\begin{array}{l}
N_{i, 0}= \begin{cases}1 & u_{i} \leq u \leq u_{i+1} \\
0 & \text { otherwise }\end{cases} \\
N_{i, k}(u)=\frac{u-u_{i}}{u_{i+k}-u_{i}} N_{i, k-1}(u)+\frac{u_{i+k+1}-u}{u_{i+k+1}-u_{i+1}} N_{i+1, k-1}(u) . \\
\frac{0}{0}=0
\end{array}\right.
$$

The exact value of the arc length of NURBS curve can be calculated with 9-points Gauss-Legendre integration formula (3),

$$
\int_{\frac{i-1}{N}}^{\frac{i}{N}} f(t) \mathrm{d} t=\frac{1}{2 N} \int_{-1}^{1} f\left(\frac{2 i-1+u}{2 N}\right) \mathrm{d} u
$$

Where $f(t)$ is integrand, $N$ is serial number of integral subinterval.

\section{Scheduling feedrate for split NURBS curve based on s ACC/DEC}

NURBS curve contains flat and sharp curve section in the ordinary course of events as shown in Fig.1 (a) trident NURBS curve. Sharp curve section with large curvatures in a NURBS curve would violate kinematic property for high-speed machining. Interpolation points where any of chord error, centripetal acceleration or jerk was violated are called critical points. Interpolation points with local maximum curvature were called cuspidal points. To find out critical points and determine their corresponding feedrates, three constraints of are considered simultaneously in Eq.(4). The critical curvature $\kappa_{c r}$ for identifying the critical points is given as [4].

$$
\kappa_{c r}=\min \left(\frac{8 \delta}{\left(v_{\max } \mathrm{T}_{s}\right)^{2}+4 \delta^{2}}, \frac{A_{n}}{v_{\max }^{2}}, \sqrt{\frac{J_{n}}{v_{\max }^{3}}}\right)
$$

Where $\delta, T_{s}, v_{\max }, A_{n}, J_{n}$ are chord tolerance, sampling time maximum feedrate, centripetal acceleration or jerk respectively. Suitable federate for cuspidal points were computed with Eq.5[5].

$$
v_{i}=\min \left(\frac{2}{\mathrm{~T}_{s}} \sqrt{\frac{1}{k_{i}^{2}}-\left(\frac{1}{k_{i}}-\delta\right)^{2}}, \sqrt{\frac{A_{n}}{k_{i}}}, \sqrt[3]{\frac{J_{n}}{k_{i}^{2}}}\right)
$$


In Eq.5, $k_{i}$ is the curvature of cuspidal points.

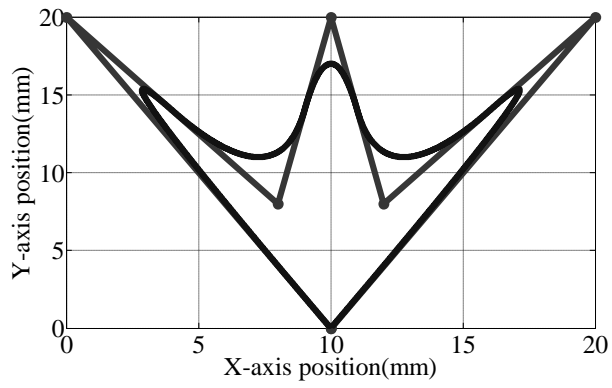

a

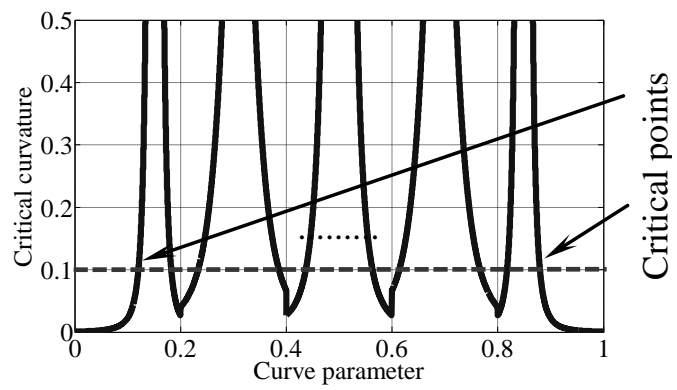

c

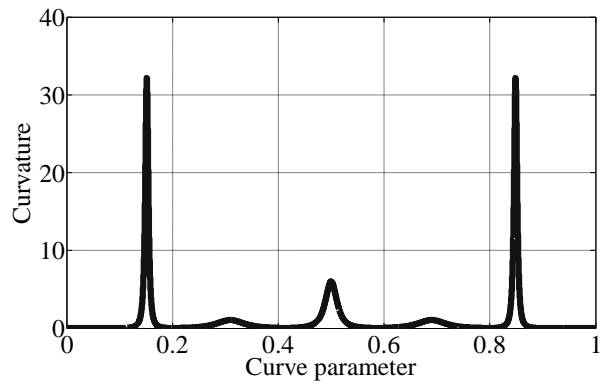

$\mathrm{b}$

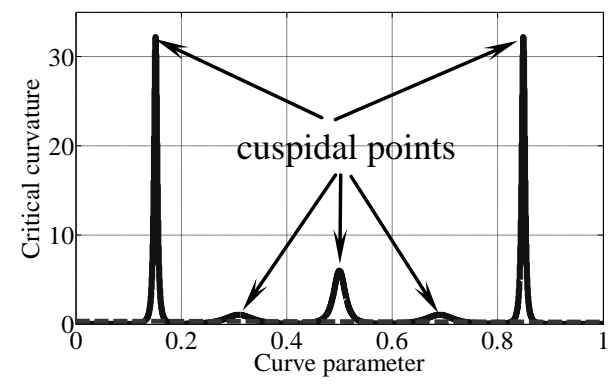

d

Fig. 1 Trident NURBS curve and its curvature. (a) Trident curve, (b) Curvature,(c) Critical points, (d)Cuspidal points

For trident NURBS curve, distribution of its curvature, critical points and cuspidal points are shown in Fig.1(b), Fig.1(c) and Fig.1(d) respectively.

After the critical points, cuspidal points were determined, the original NURBS curve was divided several sub-segments with these points, 9-points Gauss-Legendre integration formula was used to computer each one arc length respectively.The maximal federate, tangential acceleration and jerk for interpolation can be obtained from the properties of machine tools. For each cuspidal point, forward scheduling and backward scheduling method was applied according to arc length of each sub-segments, feedrate of each cuspidal points, and dynamical properties of machine tools with S ACC/DEC.

The modes of ACC/DEC was classified 21 types as start segment, stop segment, intermediate segment with/without constant acceleration with/without constant feedrate and constant federate segment according arc length of each sub-segments and dynamical properties of machine tools. Here how to determine the mode of ACC/DEC of intermediate acceleration segment was given. For intermediate segment, if $f_{e}-f_{s} \geq A^{2} / J$ is meet, and the arc length is greater than value of Eq. 6 .

$s_{i}=\frac{A}{2 J}\left(f_{s}+f_{e}\right)+\frac{f_{e}-f_{s}}{A} f_{s}+\frac{\left(f_{e}-f_{s}\right)^{2}}{2 A}$

Where $s_{i}$ is arc length of sub-segment, $A$ is tangential acceleration, $J$ is tangential jerk, $f_{s}$ is start end feedrate, $f_{e}$ is the stop end feedrate, the mode of ACC/DEC is acceleration with constant acceleration with constant feedrate. If the arc length of sub-segment is less than the value of Eq. 6 but greater than value of Eq.7,

$s_{i}=\frac{2 A}{J} f_{s}+\frac{A^{3}}{J^{2}}$

the mode of ACC/DEC is acceleration with constant acceleration without constant feedrate. If the arc length of sub-segment is less than the value of Eq.7, the mode of ACC/DEC is acceleration without 
constant acceleration without constant feedrate. For intermediate segment, if $f_{e}-f_{s} \geq A^{2} / J$ is not meet, and the arc length is greater than value of Eq.8,

$s_{3 e}=2 f_{s} \mathrm{~T}_{1}+J \mathrm{~T}_{1}^{3}$

the mode of ACC/DEC is acceleration without constant acceleration but with constant feedrate. For intermediate segment, if $f_{e}-f_{s} \geq A^{2} / J$ is not meet, and the arc length is less than value of Eq.8, the mode of ACC/DEC is acceleration is the same as the one of its arc length less than Eq.6 and Eq.7.

\section{Interpolation Case and its Analysis}

The trident curve in Fig.1(a) was used as calculation case, the control points of this curve are $(10,0)$, $(20,20),(12,8),(10,20),(8,8),(0,20)$ and $(10,0) \mathrm{mm}$. The weight of it are $[1,1,1,1,1,1,1]$, and the knot vector of it is $[0,0,0,0.2,0.4,0.6,0.8,1,1,1]$. The maximal feed $v_{\max }=100 \mathrm{~mm} / \mathrm{s}$ and acceleration $\mathrm{A}=1000 \mathrm{~mm} / \mathrm{s}^{2}$ were specified, and then the maximal jerk $\mathrm{J}=30000 \mathrm{~mm} / \mathrm{s}^{3}$ and sampling time $1 \mu \mathrm{m}$ were selected respectively considering the dynamic performance of machine tool.

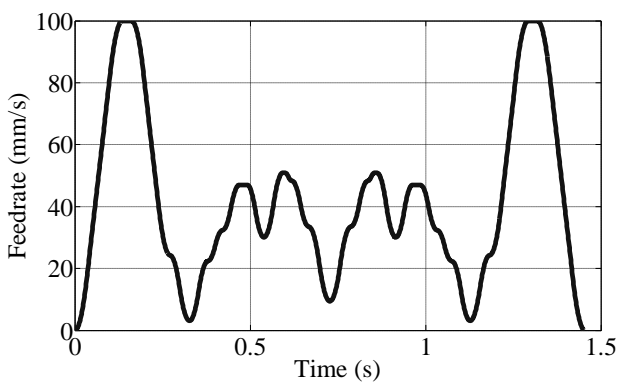

a

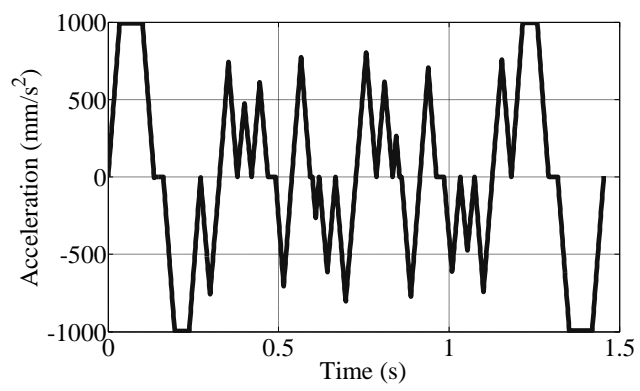

c

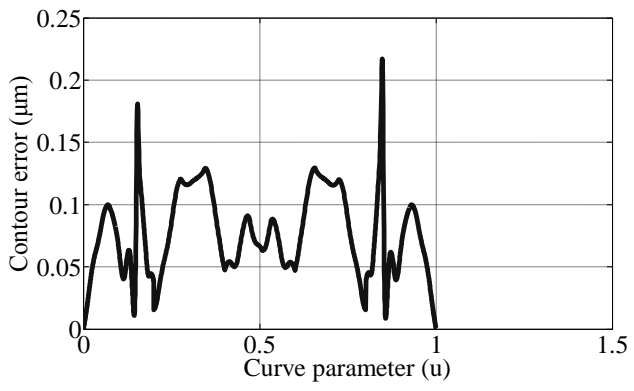

b

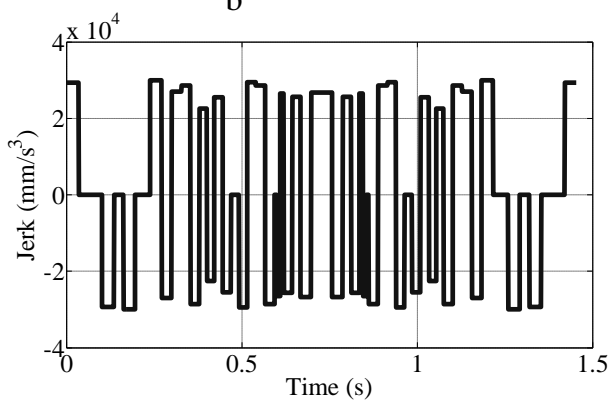

d

Fig. 2 Trident NURBS curve and its curvature. (a) Feedrate, (b) Contour error,(c)Acceleration, (d)Jerk

The interpolation feed was planned with the proposed algorithm in this paper under these kinetic characteristic parameter, the feed planning results are shown in Fig. 2 respectively. Results from Fig. 2 indicated the feed, acceleration, and jerk evolve smooth during interpolation, and the contour error is under $0.23 \mu \mathrm{m}$ with the proposed algorithm.

\section{Summary}

The NURBS curve was split several sub-segments according to cuspidal points and critical points, feedrate scanning algorithm based on $\mathrm{S}$ curve acceleration/deceleration was used to schedule the feedrate within each sub-segments and the connection points between adjacent sub-segments. The results of a interpolation indicated the proposed method not only obtain high interpolation accuracy with high interpolation feedrate, but also the acceleration and jerk evolve smooth during interpolation. 


\section{Acknowledgement}

This research was financially supported by the zhejiang provincial natural science fund LY12E05001 and the National Science Foundation 51405119.

\section{References}

[1] H. Pan, W. Yang, L. Chen et al, Adaptive piecewise NURBS curve interpolator algorithm for entireness process S-curve ACC/DEC control, China Mechanical Engineering.21,2(2010)190-195.

[2] X Du, J Huang, L-M Zhu, A complete S-shape feed rate scheduling approach for NURBS interpolator, J. Com Des Eng. (2015)1-12.

[3] M-T Lin, M-S, Tsai, H-T, Yau, Development of a dynamics-based NURBS interpolator with real-time look-ahead algorithm, Int. J. Mach Tol, 47(2007)2246-2262.

[4] A-C Lee, M-T Lin, Y-R, Pan et al., The feedrate scheduling of NURBS interpolator for CNC machine tools, Com-Aid Des. 43(2011)612-628. 